\title{
Isolated Rheumatic Pulmonary Valve Disease-Case Reports
}

\author{
Ramachandran Muthiah \\ Thoothukudi Medical College Hospital, Thoothukudi, India \\ Email: cardioramachandran@yahoo.co.uk
}

Received 4 May 2016; accepted 13 June 2016; published 16 June 2016

Copyright (C) 2016 by authors and Scientific Research Publishing Inc.

This work is licensed under the Creative Commons Attribution International License (CC BY).

http://creativecommons.org/licenses/by/4.0/

(c) (i) Open Access

\begin{abstract}
Rheumatic heart disease (RHD) is the most common cause of acquired heart disease in children and young adults worldwide and particularly developing countries continuing to experience a high incidence of this disease. The unexpected increase in the incidence of the disease in certain areas may explain the clinical and epidemiological characteristics of this disease. The key manifestation of RHD is the cardiac valvular abnormalities characterized principally by deforming the layered and avascular leaflet architecture due to inflammation and subsequent diffuse fibrosis. Mitral valve is mostly involved and pulmonary valve is rarely affected. Background of these case reports highlighted the increased incidence of rheumatic pulmonary valve disease in Thoothukudi region of India in Tamil Nadu state.
\end{abstract}

\section{Keywords}

Rheumatic Fever, Pulmonary Valve, Echocardiography, ASO Screening Test

\section{Introduction}

Rheumatic fever is the commonest form of heart disease in many developing countries of tropics and subtropics including Southern states of India. There are relatively few countries in the world without any data on rheumatic fever [1]. The information on the incidence of rheumatic fever and rheumatic heart disease (RHD) mortality is unsatisfactory because the reliable incidence data are very difficult to obtain [2] and hospital statistics give a biased picture of occurrence, since admissions depend on a host of different factors.

The literature has been reviewed repeatedly and recent reviews [3] point out that the prevalence of rheumatic heart disease in school children seems to be about 0 - 1 per thousand in developed countries, and is much higher in developing countries. Thus rheumatic heart disease prevalence rates are as high as 22 per thousand [4] and even 33 per thousand have been reported in school-age children in urban slums of some developing countries. House-to-house surveys have also been carried out, such as Berry's survey in Northern India [5]. Worldwide, 
there are only 15 million cases of rheumatic heart diseases (RHD), with 282,000 new cases and 233,000 deaths from this disease each year [6]. In contrast to trend in United States, rheumatic fever and RHD have not decreased in developing countries. Retrospective studies in developing countries showed the highest predilection for cardiac involvement and highest recurrence rate of rheumatic fever [7].

Rheumatic fever often produces a pancarditis, characterized by endocarditis, myocarditis and pericarditis. Endocarditis is manifested as valvular insufficiency and severe scarring of the valve develops during a period of months to years after an episode of acute rheumatic fever, and recurrent episodes may cause progressive damage to the valves. The mitral valve is affected most commonly and severely in $65 \%$ - $70 \%$ of patients and the aortic valve is affected in $25 \%$. The tricuspid valve is involved in only $10 \%$ of patients, almost always in association with mitral and aortic lesions. RHD is responsible to $99 \%$ of mitral valve stenosis in adults and the pulmonary valve is rarely affected.

An isolated rheumatic involvement of pulmonary valve is uncommon in literature and so it had been reported.

\section{Case Reports}

\subsection{Case- 1}

A 9 year old asymptomatic male child, having precordial murmur was referred for echocardiographic evaluation The child had 2 to 3 episodes of febrile illness with joint pains at the age of 3 - 4 years and not taken penicillin prophylaxis thereafter.. His pulse rate was $80 \mathrm{bpm}$. Blood chemistry revealed a positive ASO titer suggesting an antecedent streptococcal infection. ECG revealed the persistent juvenile pattern of T inversion in V1-V3 and $\mathrm{X}$-ray chest revealed mild prominence of main pulmonary artery. Physical examination revealed normal general appearance with a grade 3/6 systolic murmur over the precordium, most prominent in the left second intercostal space and a phasic ejection click which was loudest during expiration and diminished in intensity during inspiration. The second heart sound was soft and single. These features were consistent with valvular pulmonary stenosis. Echocardiography revealed a thickened pulmonary valve as shown in Figure 1 with commissural fusion as shown in Figure 2, moderate to severe pulmonary stenosis as in Figure 3 and a moderate pulmonary regurgitation as in Figure 4 suggesting the rheumatic pulmonary valve disease. Figure 9 revealed the pulmonary stenosis and regurgitant jets and their velocities. Figure 5 and Figure 6 revealed the normal mitral, aortic and tricuspid valves. The child is advised penicillin prophylaxis and periodic follow-up.

\subsection{Case- 2}

A 3 year old female child having grade 2/6 systolic murmur over the precordium was referred for screening echocardiopgraphy with a positive serum ASO titer. The child had a history of febrile illness during the neonatal period and the nature of illness was unknown. Pulse rate was $90 \mathrm{bpm}$. General appearance was normal and cardiac auscultation revealed 2/6 systolic murmur in the left second intercostal space. ECG and X-ray chest were normal. Transthoracic echocardiographic imaging revealed bright echoes on the tip of pulmonary valve with mild thickening suggest rheumatic inflammation as shown in Figure 7 and a mild pulmonary valve stenosis as in Figure 8. The child is advised penicillin prophylaxis and periodic follow-up.

\section{Discussion}

\subsection{Etiological Aspects}

\subsubsection{Congenital}

Diseases of the pulmonary valve are most often congenital, and only rarely due to acquired disorders such as carcinoid, rheumatic and infective endocarditis [8]. Pulmonary stenosis represents $8 \%$ - $12 \%$ of all congenital heart defects in children [9] and 15\% in adults [10]. The morphological features of pulmonary valve disease have been well described. Stenotic pulmonary valves are always anatomically abnormal (fibrous thickening and rarely calcific deposits [11]). Congenital type of pulmonary stenosis include acommissural dome-shaped, dysplastic, and bicuspid. The most common cause of isolated pulmonary valve stenosis is the congenital domeshaped acommissural valve. It is stated that stenotic pulmonary valve may be associated with some element of regurgitation. The acommissural stenotic pulmonic valve usually is a dome-shaped structure with central aperture. Ridges are usually visible that mark sites of apparently malformed commissures [12]. The second major 
type of isolated congenital pulmonary stenosis is a tricuspid pulmonary valve in which all the three cusps are greatly thickened and rubbery. This form of congenital abnormal pulmonary valve has been termed "dysplastic pulmonic stenosis" [13]. In such dysplastic valves, there usually is no commissural fusion but the valve annulus is small and histologically, the valve cusps contain extensive amount of acid mucopolysaccharide-stained material and fibrous tissue and lack of poststenotic dilatation of the pulmonary artery. The pulmonary valves in patients with Noonan's syndrome characterized by “moon's facies” are genetically dysplastic. In patients with Tetralogy of Fallot, pulmonic valve stenosis is frequently associated with bicuspid pulmonary valve. All congenital forms had thickened cusps with (bicuspid, tricuspid) commissural fusion [14] or without (domed, unicommissural, dysplastic).

\subsubsection{Rheumatic}

Rheumatic disease causing pulmonary valve stenosis is quite uncommon and, when it occurs, it is invariably associated with rheumatic disease of other cardiac valves. Vela and colleagues [15] reported frequent and more severe pulmonic valve lesions in patients with rheumatic heart disease in Mexico city. These authors suggested that pulmonary hypertension associated with the altitude of Mexico city, increased the stress on the pulmonary valve and making it more prone to damage. Rheumatic forms also had thickening and commissural fusion which are more evident at leaflet tips and subvalvular apparatus.

Isolated rheumatic involvement of pulmonary valve is observed in children $<15$ years of age at the tropical zone of Thoothukudi in India. Majority are presented with mild to moderate pulmonic stenosis without the involvement of other cardiac valves. Earliest rheumatic involvement of the pulmonic valve is noticed frequently among school referral cases in this region.

\subsection{Screening Test}

Rheumatic fever is a non-suppurative sequelae to the rheumatogenic strains of Lancifield group A $\beta$ hemolytic streptococcal infection of tonsillopharynx after a latent period of approximately 3 weeks and causing exudative and proliferative inflammatory reaction which in turn damage to collagen fibrils and ground substance of connective tissue. These rheumatogenic strains are often encapsulated mucoid strains, rich in $\mathrm{M}$ proteins and resistant to phagocytosis. These strains are strongly immunogenic and immunologic cross-reactions between the streptococcal carbohydrate and valvular glycoprotein, resulting damage to heart valves. Group A streptococci (GAS) elaborate the cytolytic exotoxins, streptolysins $\mathrm{S}$ and $\mathrm{O}$ that act as antigens and the affected individuals produce specific antibodies against these antigens. Of these two toxins, streptolysin $\mathrm{O}$ induces persistently high antibody titers that provide a useful marker of GAS infection and its nonsuppurative complications. The antibodies to the extracellular streptococcal antigens rise during the first month after infection and then plateau for 3 6 months before returning to normal levels after $6-12$ months. When the ASO titer peaks ( 2 - 3 weeks after the onset of rheumatic fever), the sensitivity of the test is $80 \%-85 \%$. Several serological tests are now available to determine the occurrence of an antecedent streptococcal infection. Most of these tests assay for neutralizing antibodies to streptococcal extracellular enzymes. The first such test, described by Todd in 1932 [16] determines the titers of serum antibodies that neutralize streptolysin O. This test, the anti-streptolysin O (ASO) test, is still the most standardized and universally used of the streptococcal antibody tests. ASO titers may vary with age, geographic area, and other factors influencing the frequency of streptococcal infection. Titers of 200 to 300 units/ml are common in healthy children 5 to 14 years of age who live in crowded cities at the temperate zone of United States. Surveys of healthy school children 6 - 10 years of age, found anti-streptolysin-O titers $>200$ Todd units in $15 \%$ - 70\% of children [17]. ASO titers greater than 200 to 250 Todd units per $\mathrm{ml}$ are generally considered elevated. Titers $>333$ Todd units in children and $>200$ Todd units in adults are taken as positive for streptococcal infection. In this region of Thoothukudi, titers $>200 \mathrm{IU} / \mathrm{ml}$ is taken as positive for both adults and children.

Serum ASO titer (a non-type-specific antibody test) was positive in these children, suggesting the laboratory evidence of antecedent Group A streptococcal infection.

\subsection{Echocardiography}

In individuals with rheumatic heart disease (RHD), echocardiography is useful to identify and quantify the valvular lesions. Studies in Cambodia and Mozambique have demonstrated a 10-fold increase in the prevalence of 
RHD when echocardiography is used for clinical screening compared with strictly clinical findings [18]. In individuals with chronic RHD, echocardiography tracks the progression of valve stenosis and may help to determine the time for surgical intervention. The leaflets of affected valves become thickened diffusely, with fusion of commissures and chordae tendineae. Increased echo density of the valve may signify calcification. World Heart Federation has published guidelines to identify individuals with RHD without a clear history of acute rheumatic fever. Based on 2D and color Doppler imaging, patients are divided into 3 categories_-definite, borderline and normal. For pediatric patients (defined as age $<20$ years), definite echo features include pathological regurgitation and at least two morphological features of rheumatic involvement of the valve such as commissural fusion, leaflet thickening, calcification and restrictive mobility of the valve.

Most children with pulmonary stenosis do not require evaluation beyond echocardiography. Two dimensional and Doppler imaging is the sine qua non of its diagnosis and a thickened pulmonary valve with restricted systolic motion (doming) in the parasternal short axis view is the characteristic feature [19] as shown in Figure 1. The main pulmonary artery is dilated in almost all cases as shown in Figure 1 and Figure 2. This dilatation is independent of the severity of the pulmonary valve obstruction and presumably related to a high-velocity jet across the stenotic valve [20]. The commonly occurring dilatation of the main pulmonary artery distal to the stenotic orifice (post stenotic dilatation) is remarkably absent in patients with dysplastic pulmonary valve, characterized by markedly thickened, nodular, and immobile valve leaflets. Pulmonary valve annular measurements is useful in selecting the diameter of the balloon to be used during valvuloplasty and can be compared with normal values to determine if the annulus is hypoplastic.

Color Doppler imaging shows smooth, laminar subpulmonary flow (blue) and some flow acceleration (red) immediately beneath the pulmonary valve with turbulent (mosaic) flow beginning immediately distal to the pulmonary valve leaflets as shown in Figure 3. Doppler studies can be used to accurately determine the velocity of flow at single or multiple levels, which then can be converted to reproducible pressure gradients by applying the modified Bernoulli equation-pressure gradient $(\mathrm{mmHg})=4 \times \mathrm{V}^{2}(\mathrm{~m} / \mathrm{s})$ [21]. The sample volume moved across the pulmonary valve demonstrates an abrupt increase in peak Doppler flow velocity, which suggests pulmonary valve obstruction as shown in Figure 9. A gradients of $>50 \mathrm{mmHg}$, as diagnosed using a continuous-wave Doppler recording through the pulmonary valve indicate severe stenosis. This Doppler measurement represent peak instantaneous gradient which overestimates the peak-to-peak systolic catheterization gradient, presumably because of a pressure-recovery phenomenon [22]. In this child, the peak Doppler flow velocity of pulmonary stenotic jet $3.45 \mathrm{~m} / \mathrm{s}$ as shown in Figure 9 and the gradient $48 \mathrm{mmHg}$ suggest a moderate to severe pulmonary stenosis. Pulmonary regurgitation is easily seen during Doppler imaging but is unlikely to be present without previous surgical or balloon valvuloplasty in valvular pulmonary stenosis.

Valvular thickening has been demonstrated to be a feature of RHD on echocardiography and is often most marked at leaflet tips [23]. The thickened pulmonary valve with commissural fusion as shown in Figure 1 and Figure 2, a moderate to severe pulmonary stenosis as in Figure 3, associated with moderate pulmonary regurgitation as in Figure 4 with a positive ASO titer indicating the antecedent streptococcal infection, suggesting the rheumatic pulmonary valve disease in this male child aged 9 years.

Figure 7 illustrate bright echo density on the tips of the pulmonary valve suggest a rheumatic inflammation associated with mild pulmonary valve stenosis as in Figure 8 in a 3-year old female child.

\subsection{Treatment}

Therapy is directed towards preventing recurrent rheumatic heart disease in children and monitoring for the complications and sequelae of chronic rheumatic heart disease in adults. The importance of preventing recurrences of rheumatic fever is evident. The incidence of residual rheumatic heart disease at ten years is 34\% in patients without recurrence and $60 \%$ in patients with recurrent rheumatic fever. Disappearance of the murmur, when it occurs, happens within 5 years in $50 \%$ of patients. Thus, significant number of patients experience resolution of valve abnormalities even 5 - 10 years after the episode of rheumatic fever. Oral penicillin V remains the drug of choice for treatment of streptococcal pharyngitis. Oral penicillin $250 \mathrm{mg}$ for children and $500 \mathrm{mg}$ for adults, given 3 times daily for 10 days is the primary recommended regimen. For recurrent pharyngitis, a second 10-day course of the same antibiotic may be repeated. GABHS (Group A beta hemolytic streptococcus) carriage is difficult to eradicate with conventional penicillin therapy. Thus, oral clindamycin (20 mg/kg/day) in 3 divided doses for 10 days is recommended. Although oral penicillin prophylaxis is also effective to prevent recurrent 


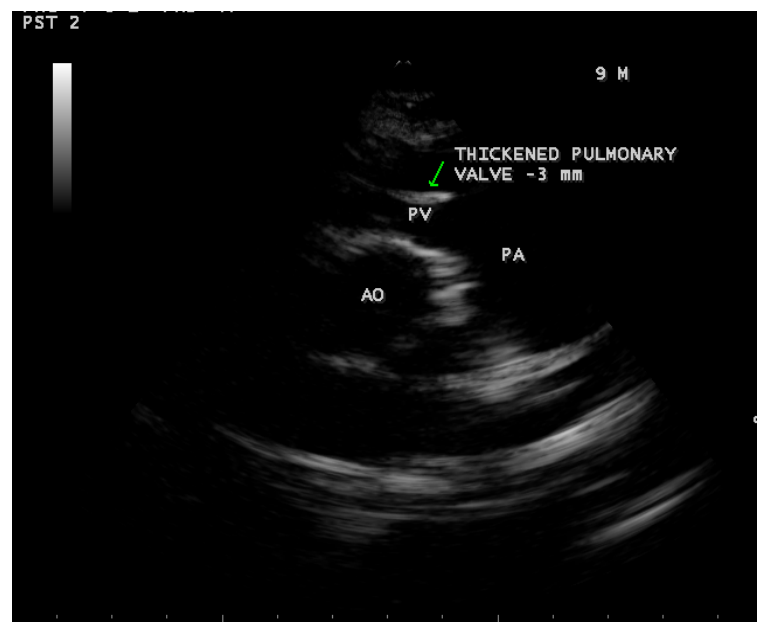

Figure 1. Short axis view showing the thickened pulmonary valve, well seen at leaflet tips, AO-aorta, PA-pulmonary artery, PV-pulmonary valve.

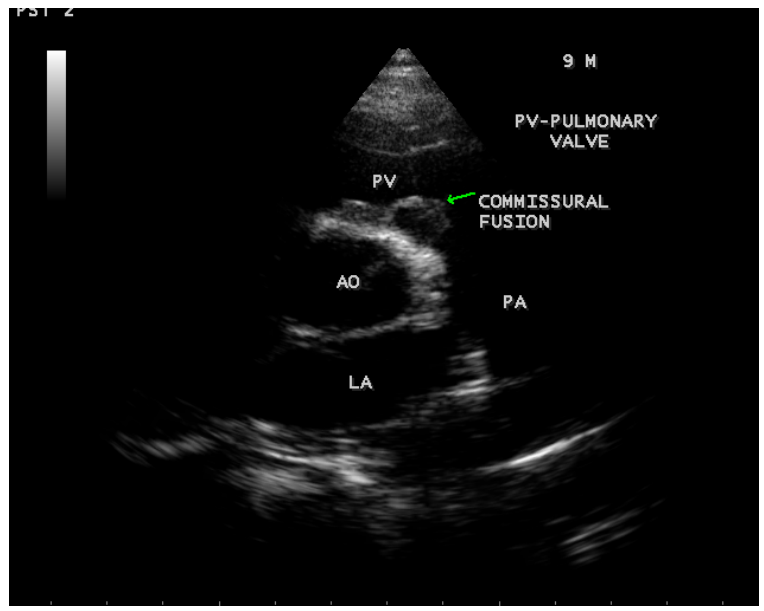

Figure 2. short axis view showing the commissural fusion of pulmonary valve, AO-aorta, PV-pulmonary valve, LA-left atrium, PA-pulmonary artery.

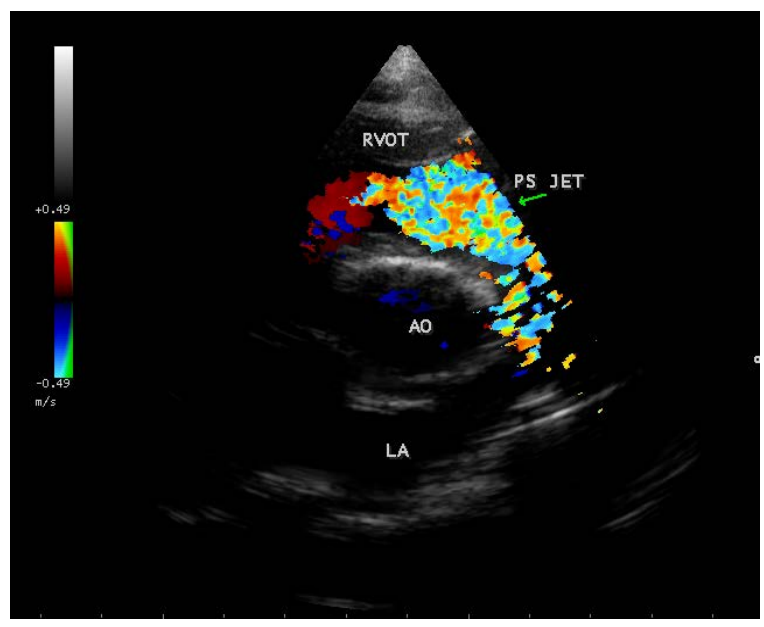

Figure 3. Short axis view-color Doppler imaging showing pulmonary stenosis jet, AO-aorta, PS-pulmonary valve stenosis, LA-left atrium, RVOT-right ventricular outflow tract. 


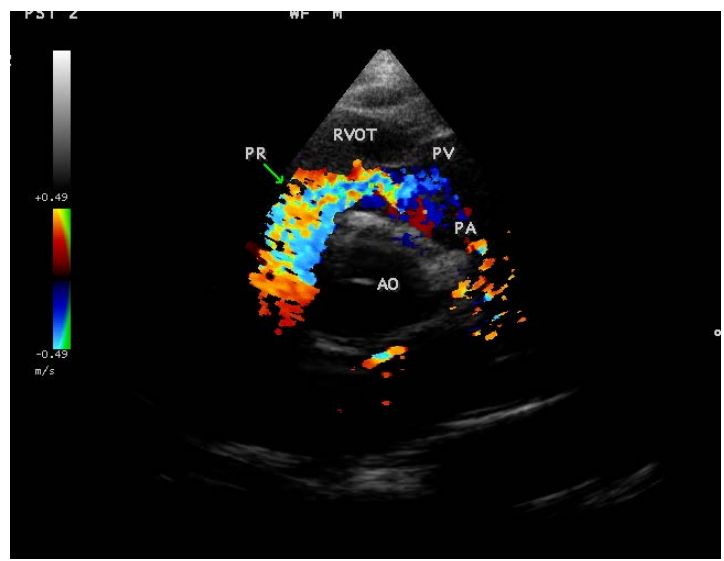

Figure 4. Short axis view-color Doppler imaging showing pulmonary regurgitant jet, AO-aorta, PR-pulmonary valve regurgitation, PV-pulmonary valve, PA-pulmonary artery, RVOT-right ventricular outflow tract.

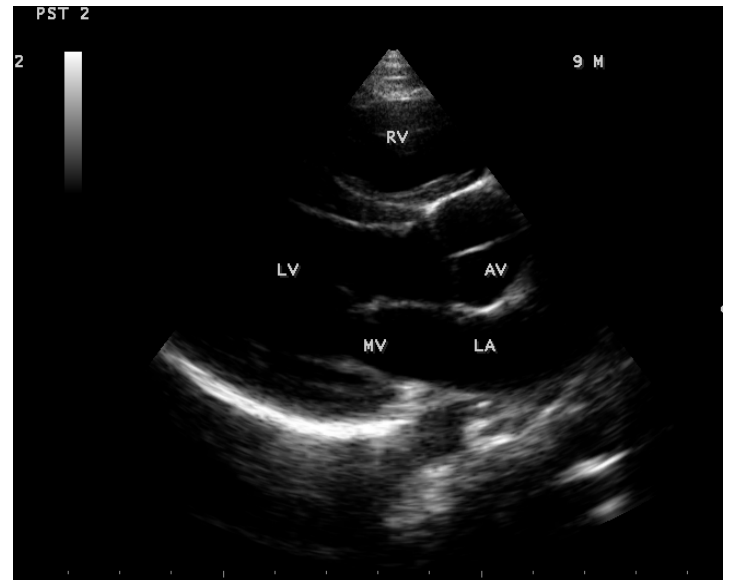

Figure 5. Parasternal lomg axis view showing the normal mitral and aortic valves. LA-left atrium, LV-left ventricle, MV-mitral valve, AV-aortic valve, $\mathrm{RV}$-right ventricle.

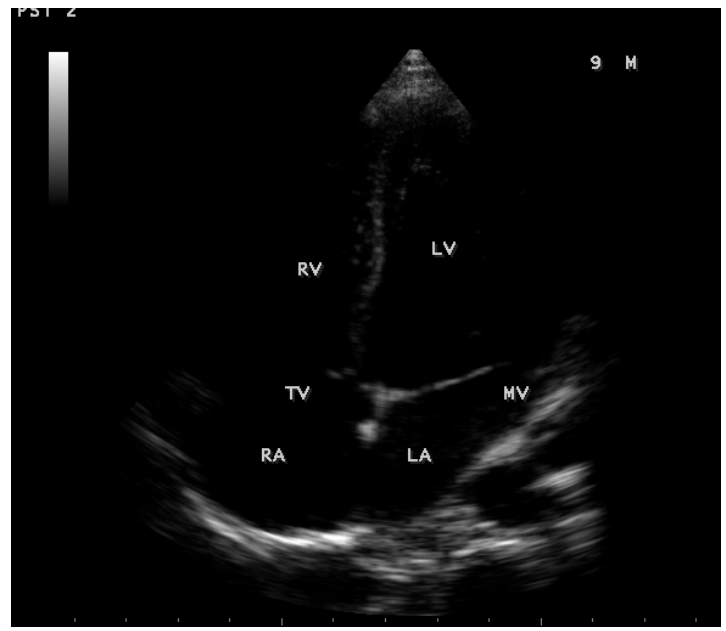

Figure 6. Apical four chamber view showing the normal mitral and tricuspid valves, MV-mitral valve, TV-tricuspid valve, LA-left atrium, RA-right atrium, $\mathrm{LV}$-left ventricle, $\mathrm{RV}$-right ventricle. 


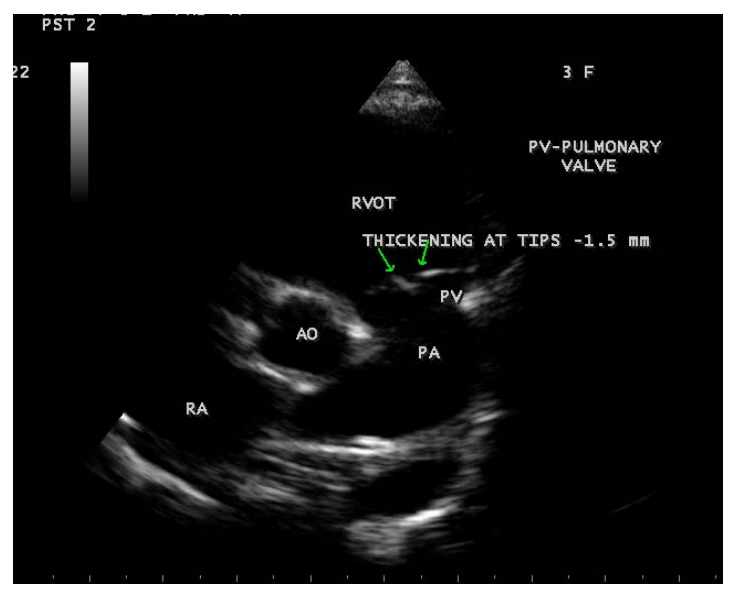

Figure 7. Short Axis view showing bright echoes on the tips of pulmonary valve suggesting Rheumatic inflammation, AO-aorta, PA-pulmonary artery, PV-pulmonary valve, RA-right atrium, RVOT-right ventricular outflow tract.

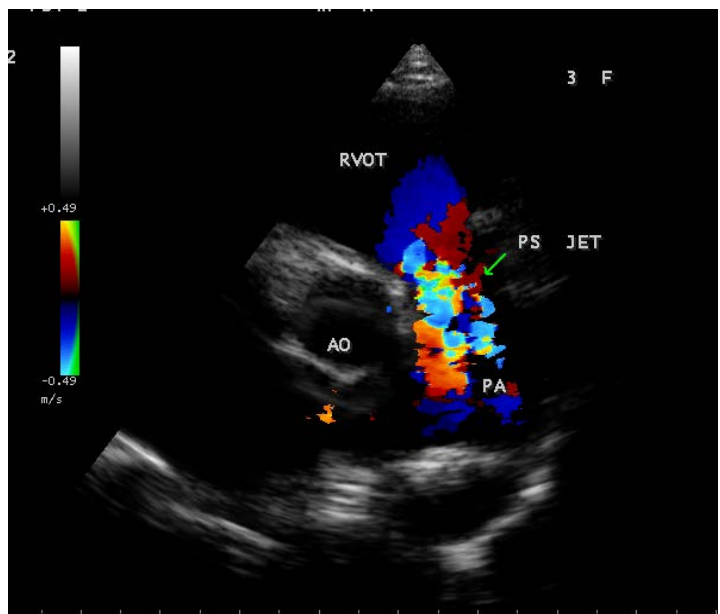

Figure 8. Short axis view showing the pulmonary stenosis jet, AO-aorta, PApulmonary artery, PS-pulmonary valve stenosis, RVOT-right ventricular outflow tract.

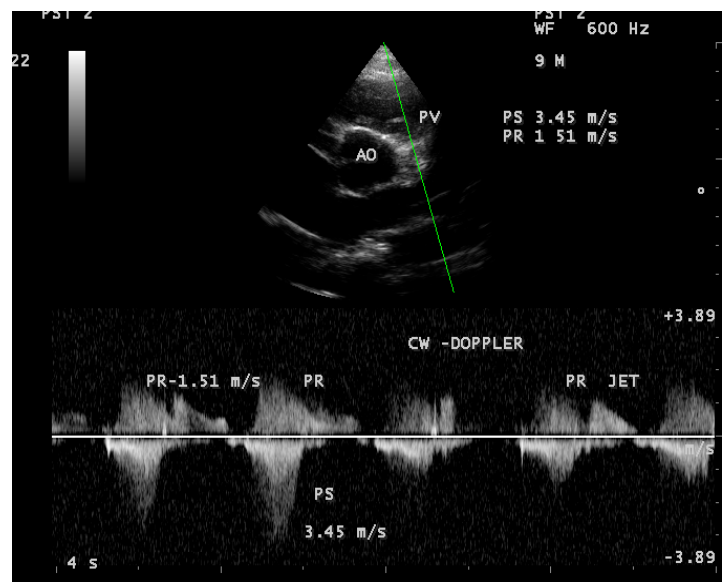

Figure 9. Continuous Wave (CW) Doppler imaging showing the velocities of pulmonary stenosis and regurgitation, AO-aorta, PS-pulmonary valve stenosis, PR-pulmonary valve regurgitation, RVOT-right ventricular outflow tract. 
episodes, data from the World Health Organization (WHO) indicate that the recurrence rate of GABHS pharyngitis is lower when penicillin is administered parentally.

In case-1, an injection of 1.2 million units of benzathine penicillin $G$ intramuscularly every 3 weeks for this 9-year old boy and in case-2, 0.6 million units for this 3-year old female child are advised as a preventive and prophylactic therapy for a duration well into adulthood and preferably for life. Tompkin et al. [24] reported that signs of rheumatic valvular disease resolve in $70 \%-80 \%$ of patients with rheumatic carditis who adhere to prophylaxis. Thus, emphasis should be placed on the importance of prophylaxis in affording a good prognosis for recovery even in patients with severe heart disease and prevention of recurrence to reduce cardiac morbidity from this disease.

In acute rheumatic heart disease, heart catheterization is not indicated. With chronic disease, heart catheterization has been performed to evaluate the valve disease and to balloon the stenotic valve. More recently, the interventional procedures are increasingly performed under general anaesthesia and the gradients are usually lower than those with conscious sedation. Therefore, the Doppler-echocardiography gradients should be used in making the decision regarding balloon pulmonary valvuloplasty and balloon dilation should be performed only in patients with peak-to-peak gradient of more than $50 \mathrm{mmHg}$. Patients with echocardiographic evidence of clinically significant pulmonary stenosis (50 - $60 \mathrm{mmHg}$ ) should undergo diagnostic and therapeutic cardiac catheterization with preparation for balloon dilation of the pulmonary valve [25].

The positive ASO titers of these cases indicate the recent streptococcal infection suggesting the recurrent episodes, both preventive and prophylactic therapies are indicated A peak gradient of $48 \mathrm{mmHg}$ (PS velocity - 3.45 $\mathrm{m} / \mathrm{s}$ ) across the pulmonary valve suggesting moderate to severe stenosis and a pulmonary diastolic pressure of 9 $\mathrm{mmHg}$ (PR velocity - $1.51 \mathrm{~m} / \mathrm{s}$ ) on Doppler-echocardiography which is similar to catheter-based pressure in the pulmonary veins (PCWP = LA pressure) suggest an isolated pulmonary valve disease of rheumatic etiology as shown in Figure 9. Adherence of prophylaxis may resolve valve damage and so these patients are advised periodic follow up. Since there is a significant pulmonary regurgitation associated with stenosis in case-1, balloon valvuloplasty is not preferred at this moment.

\subsection{Echocardiographic Screening}

Handheld echocardiography has been investigated as a screening tool and found to be $90 \%$ sensitive and $92 \%$ specific for identifying patients with rheumatic heart disease in Uganda children [26]. A study by Goodwin et al. assessed the value of handheld echocardiography over auscultation to identify rheumatic heart disease. The study found that auscultation alone is a poor screening test for rheumatic heart disease and that handheld echocardiography significantly improves detection of rheumatic heart disease in resource-limited settings [27].

\section{Conclusion}

Rheumatic involvement of pulmonary valve is frequently observed in the coastal district of Thoothukudi in India and an isolated rheumatic pulmonary valve disease in a 9 year old male and in a 3 year old female child is detected by Transthoracic echocardiographic imaging. Both echocardiography and ASO screening test, which are adapted to detect rheumatic cases and early administration of penicillin prophylaxis is practiced in positive cases as a preventive measure and penicillin therapy is given to treat any episodes of streptococcal pharyngitis which is endemic in this tropical region. From these case reports, it is known that a possibility of rheumatic pulmonary valve disease is more common similar to congenital etiology and more frequent like rheumatic mitral, aortic and tricuspid valve diseases in this region.

\section{References}

[1] Strasser, T. (1976) Rheumatic Fever and Rheumatic Heart Disease in The 1970's. Public Health Reviews, 5, 207.

[2] Spector, A. (1968) Measurement of the Incidence of Acute Rheumatic Fever, a Methodological Study. American Journal of Public Health, 58, 1950. http://dx.doi.org/10.2105/AJPH.58.10.1950

[3] Quinn, R.W. (1873) Rheumatic Fever and Rheumatic Heart Disease. Public Health Reviews, 2, 155.

[4] Gharagozloo, R.A., et al. (1972) Streptococcal Infection. Rheumatic Fever and Rheumatic Heart Disease among 500 Jewish School Children in Teheran. Israel Journal of Medical Sciences, 8, 18.

[5] Berry, J.N. (1972) Prevalence Survey for Chronic Rheumatic Heart Disease and Rheumatic Fever in Northern India. 
British Heart Journal, 34, 143. http://dx.doi.org/10.1136/hrt.34.2.143

[6] Seckeler, M.D. and Hoke, T.R. (2011) The Worldwide Epidemiology of Acute Rheumatic Fever and Rheumatic Heart Disease. Clinical Epidemiology, 3, 67-84. http://dx.doi.org/10.2147/CLEP.S12977

[7] Orun, U.A., Ceylan, O., Bilici, M., Karademir, S., Ocal, B. and Senocak, F. (2012) Acute Rheumatic Fever in the Central Anatolia Region of Turkey. A 30-Year Experience in a Single Center. European Journal of Pediatrics, 171, 361368.

[8] Rao, P.S., Alpert, J.S., Dalen, J.E. and Rahimtoola, S. (2000) Pulmonary Valve Disease. Valvular Heart Disease, 3rd Edition, Lippencott Raven, 339-376.

[9] Nadas, A. and Fyler, D.C. (1992) Pulmonary Stenosis. Nadas’ Pediatric Cardiology, Hanley \& Belfus, Philadelphia, 459-470.

[10] Johnson, L.W., Grossman, W., Dalen, J.E. and Dexter, L. (1972) Pulmonic Stenosis in the Adult. Long-Term Follow up Results. New England Journal of Medicine, 287, 1159-1163.

[11] Roberts, W.C., Mason, D.T., Morrow, A.G. and Braunwald, E. (1968) Calcific Pulmonic Stenosis. Circulation, 37, 973-978. http://dx.doi.org/10.1161/01.CIR.37.6.973

[12] Waller, B.F. (1984) Morphological Aspects of Valvular Heart Disease, Part II. Current Problems in Cardiology, 9, 174. http://dx.doi.org/10.1016/0146-2806(84)90013-6

[13] Koretzky, E.D., Moeller, J.H., Koms, M.E., Schwartz, C.T. and Edwards, J.E. (1969) Congenital Pulmonary Stenosis Resulting from Dysplasia of Valve. Circulation, 40, 43-48. http://dx.doi.org/10.1161/01.CIR.40.1.43

[14] Gikonyo, B.M., Lucas, R.V. and Edwards, J.E. (1987) Anatomic Features of Congenital Pulmonary Valvar Stenosis. Pediatric Cardiology, 8, 109-116. http://dx.doi.org/10.1007/BF02079465

[15] Vela, J.E., Contreras, R. and Sosa, F.R. (1969) Rheumatic Pulmonary Valve Disease. American Journal of Cardiology, 23, 12-18. http://dx.doi.org/10.1016/0002-9149(69)90236-7

[16] Todd, E.W. (1932) Anti Haemolysin Titers in Haemolytic Streptococcal Infections and Their Significance in Rheumatic Fever. British Journal of Experimental Pathology, 13, 248-259.

[17] Taranta, A. and Markowitz, M. (1989) Rheumatic Fever. Kluwer Academic Publishers, Boston, 1-18. http://dx.doi.org/10.1007/978-94-009-1261-8 1

[18] Marijon, E., Qu, P., Celermajer, D.S., Ferreira, B., Mocumbi, A.O., Jani, D., et al. (2007) Prevalence of Rheumatic Heart Disease Detected by Echocardiography Screening. New England Journal of Medicine, 357, 470-476. http://dx.doi.org/10.1056/NEJMoa065085

[19] Weyman, A.E, Hurwitz, R.A., Girod, D.A., Dillon, J.C., Feigenbaum, H. and Green, D. (1977) Cross-Sectional Echocardiographic Visualization of the Stenotic Pulmonary Valve. Circulation, 56, 769-774. http://dx.doi.org/10.1161/01.CIR.56.5.769

[20] Rodbard, S., Ikeda, K. and Montes, M. (1967) An Analysis of Mechanisms of Post Stenotic Dilatation. Angiology, 18, 349-367. http://dx.doi.org/10.1177/000331976701800601

[21] Armstrong, W.F. and Ryan, T. (2001) Feigenbaum’s Echocardiography. In: Lilly, L.S., Eds., Braunwald Heart Disease, 6th Edition, Chapter 7, Saunders, New York, 170.

[22] Singh, G.K., Balfour, I.C., et al. (2003) Lesion Specific Pressure Recovery Phenomenon in Pediatric Patients. A Simultaneous Doppler and Catheter Correlative Study. Journal of American College of Cardiology, 41, 493. http://dx.doi.org/10.1016/S0735-1097(03)82689-X

[23] Naito, H., Marganroth, J., Mardelli, T.J., Chen, C.C. and Driefus, L.S. (1980) Rheumatic Mitral Stenosis, Cross-Sectional Echocardiographic Analysis. American Heart Journal, 100, 34-40. http://dx.doi.org/10.1016/0002-8703(80)90276-8

[24] Tompkins, D.G., Boxerbaun, B. and Liebman, J. (1972) Long-Term Prognosis of Rheumatic Fever Patients Receiving Regular Intramuscular Benzathine Penicillin, Circulation, 45, 543-551. http://dx.doi.org/10.1161/01.CIR.45.3.543

[25] Rao, P.S. (1988) Indication for Balloon Pulmonary Valvuloplasty. American Heart Journal, 116, 1661-1662. http://dx.doi.org/10.1016/0002-8703(88)90790-9

[26] Beaton, A., Aliku, T., Okello, E., Lubega, S., McCarter, R. and Lwabi, P. (2014) The Utility of Handheld Echocardiography for Early Diagnosis of Rheumatic Heart Disease. Journal of American Society of Echocardiography, 27, 42-49. http://dx.doi.org/10.1016/j.echo.2013.09.013

[27] Godown, J., Lu, J.C., Beaton, A., Sable, C., Mirembe, G., Sanya, R., et al. (2015) Handheld Echocardiography versus Auscultation for Detection of Rheumatic Heart Disease. Pediatrics, 135, e939-e944. http://dx.doi.org/10.1542/peds.2014-2774 